\title{
Infection Control and Prevention Strategies in Dental Practices during the Covid-19 Pandemic
}

\author{
Annisaa Dayu Sinatrya ${ }^{\mathrm{a}}$, Arlina Dewi ${ }^{\mathrm{b}}$ \\ amail: dayuannisaa@gmail.com \\ ${ }^{a}$ Student of Hospital Administration Management Study Program, Muhammadiyah University of Yogyakarta \\ ${ }^{b}$ Lecturer of Hospital Administration Management Study Program, Muhammadiyah University of Yogyakarta
}

\begin{abstract}
The coronavirus-19 (COVID-19) pandemic caused by Coronavirus is causing severe acute respiratory infections. The profession of dental health service workers must know the proper infection prevention and control. The profession of dental health care workers in low-middleincome countries is at odds with developed countries in facing the challenges of a lack of personal protective equipment (PPE) and infrastructure constraints. This study aims to determine infection prevention and control strategies in dental health service practices during the Covid-19 pandemic in lower middle countries. The method used in this paper is a systematic literature review method and is analyzed according to inclusion and exclusion criteria, carried out by reviewing journals traced from international databases published between 2019 and 2021.Researchers conducted a search for data sources from the Pubmed and Mendeley databases. Search for articles in 2019-2021. There are 20 journals that fall into the criteria. The results showed that during the Covid-19 pandemic, infection prevention and control measures were needed in dental service practices in middle- and lower-income countries. Dental treatment procedures should be carried out with cross infection prevention and control in accordance with procedures and guidelines such as; patient screening, pre-treatment, treatment, post-treatment, and prevention in the dental health service practice room
\end{abstract}

Keywords: COVID-19; Dental; infection prevention and protection middle- and lower-income countries.

\section{Introduction}

The 2019 Coronavirus disease (COVID 19) outbreak began in Wuhan, China, last December and has become a major public health problem not only in China but also in countries around the world. On January 30, 2020, the World Health Organization (WHO) announced that this outbreak had caused a public health emergency of international concern (Mahase, 2020). The novel Coronavirus was originally named 2019-nCoV and is officially known as Coronavirus 2 (SARS CoV-2) severe acute respiratory syndrome. As of February 26, COVID-19 has been recognized in 34 countries, with a total of 80,239 laboratory confirmed cases and 2,700 deaths (WHO 2020). An acute respiratory disease caused by a previously unknown beta-coronavirus emerged in the city of Wuhan, Hubei province, and spread rapidly across China on February 11, the International Commission on Classification of Viruses declared the virus named severe acute respiratory syndrome coronavirus-2 (SARS). - CoV-2) and, on the same day, 
WHO announced the disease caused by the infection called the 2019 coronavirus disease (COVID-19) (Wang et al., 2020).

Corona virus disease 2019 (COVID-19) is spreading rapidly to various countries. On March 2, 2020, the first case of COVID-19 was found in Indonesia (Kemenkes RI, 2020). As of March 31, 2020, according to data from the Ministry of Health, there were 1,528 confirmed cases of COVID-19 in Indonesia and 136 deaths related to the disease. The national case fatality rate (CFR) is also much higher than that of the PRC (8.9\% vs 4\%). Health facilities in Indonesia are not ready to deal with COVID-19. The massive preparations should have been taken seriously at the start of the spread of the disease in the People's Republic of China. A preparedness plan must be prepared by ensuring the availability of medicines, personal protective equipment (PPE) and human resources needed to deal with a global outbreak. According to the latest data from the Indonesian Ministry of Health, there are only 309,100 hospital beds in Indonesia, most of which are located on the island of Java. Additionally, there are less than 6,000 Intensive Care Unit (ICU) beds across the country. The numbers seem overwhelming, but in reality, Indonesia only has 2.7 ICU beds per 100,000 population and is thus among the lowest in Asia. In addition, mechanical ventilators are not widely available in rural areas and there is a shortage of protective equipment. For health workers, mass screening was selected by the State Palace to be carried out in March 2020. At the end of March 2020, the President of Indonesia finally decided to implement large-scale social restrictions / large-scale social restrictions in cities and provinces, not in the regions quarantine. Regional quarantine is one of the four types of health quarantine according to the 2018 Health Quarantine Law. The government also emphasizes the need to stay at home for all Indonesian citizens.

The toughest health challenges are currently occurring due to the spread of the COVID-19 virus in more than 200 countries. The virus can spread rapidly more contagiously than severe acute respiratory syndrome coronavirus.

The most common symptoms of COVID-19 are fever and cough, headache, hemoptysis and diarrhea. In addition, two main symptoms related to mouth, dry mouth and amblygeustia, have been found in relatively high COVID-19 patients, suggesting that oral symptoms can also be considered as early symptoms of COVID-19 infection (Wang et al., 2020).

The protocol of social distancing requires individuals to limit groups and maintain physical distance. Some countries that have implemented social distance show an influence on the spread of this disease. Evidence from Singapore, China, and South Korea shows a decrease in the rate of transmission with the application of social distance. Social distance is essential for COVID-19. People infected with the COVID-19 virus are reported to experience asymptomatic for up to 14 days resulting in a rapid spread of the virus. As a result, some health workers recommend self-quarantine for 2 weeks after possible exposure to the virus. These changes have the potential to increase emergencies related to visits to dental service practices and further contribute to greater transmission of the virus (Long et al., 2020). During the COVID-19 pandemic, the incidence of dental disease will continue. However, to prevent the spread of this deadly respiratory disease is likely to lead to reduced access to treatment due to restrictions on the activities of the population (Long et al., 2020). 
During the COVID-19 pandemic, both dental patients and dental health workers were at high risk of being infected in practice. The dentist's profession is inseparable from the possibility to come into direct and indirect contact with microorganisms in the patient's blood and saliva so that the spread of this infection can occur. This leads to action in dentist practice putting dentists at high risk of infectious diseases(Khader $\mathrm{Y}$ et al, 2020). Furthermore, dentists experience challenges in preventing transmission due to frequent procedures that produce aerosols (Long et al., 2020). This causes the practice room and various surfaces of the dental device to be contaminated, thus increasing the risk of infection for dental healthcare workers and subsequent dental patients (Peng et al., 2020). To reduce the risk of crossinfection between patients and dental health workers, infection control measures are needed to protect patients and dental health workers (Prospero, Savini and Annino, 2003).

Clinical protocols need to be established to be applied in the work environment to avoid new infections and progressive spread of the virus. Clinically daily practice, the patient's oral fluids, contamination of materials, and the surface of the dental unit can act as a source of transmission both for dentists and assistants, and for the patient himself. Saliva and blood droplets that settle on the surface or inhalation of aerosols produced by rotating instruments and ultrasonic handheld devices are a risk to those who occupy or will occupy such environments. Therefore, the use of disinfectants and personal protective equipment (PPE) remains essential for the proper development of the dental profession (Luzzi et al., 2020).

Covid-19 transmission in hospitals between patients and healthcare providers has been widely reported. A strategy is needed to reduce the spread of COVID-19 in healthcare settings.Studies in the United States and high-income countries (HIC) have shown that the risk of relating to transmission in hospitals is low when infection control measures are always applied. In low and middle income countries (LMIC) with limited availability of resources and limited health staff can pose challenges due to the increasing cases of COVID-19. Recommendations to minimize the risk of spreading nosocomial infections in resource-limited facilities in low- and middle-income countries (LMIC) are urgently needed. The review of Cochrane 2020 found that the lack of adequate isolation rooms was also considered a major barrier to IPC practice by healthcare workers, particularly in LMIC countries.(Cobb et al., 2021).

A study shows that the existence of a practice closure is also related to the level of a country. LMIC countries with HIC countries with better health care systems may have a better capacity to manage COVID-19 complications, resulting in lower mortality, panic and anxiety rates, and less chance of closing dentists. In this study found that there are fewer private practice closures in HIC than in LMIC.This is because HIC provides financial support for dental practices to avoid closure due to economic losses by offering funds, loans, and credits to help pay salaries and supplies. In addition, dentists at HIC also generally have higher per capita incomes, which means better financial stability despite declining incomes. In LMIC countries there are no reports of plans for economic support to assist the dental sector in meeting the needs of practice(Abdelrahman et al., 2021).

The challenge at LMIC is ensuring the availability of adequate PPE for use by visitors and ensuring adequate instruction and supervision regarding hand hygiene, proper use of PPE, and attention to surface contamination can also pose challenges. In a study of health facilities in Tanzania, adherence to 
hand hygiene and disinfection practices was low among healthcare workers. Lack of compliance by health workers can increase health risks related to infection transmission (Cobb et al., 2021).

The global burden of oral health disease has doubled in both developed and developing countries. There is great concern that dental care services are inaccessible to most of the population due to rising medical costs. In most developing countries this situation occurs because the health care is not well regulated and is not even considered as an important component of the primary health care system (Pethani, Allana and Hussain, 2020).

Compliance with guidelines and regulations is determined not only by the availability of various personal protective equipment (PPE) but their affordability and availability, especially in middle- and lower-income countries, such as South Africa (McMahon et al., 2020). Moreover, the situation is further complicated by the fact that dental care is not well regulated in this country and is not even considered one of the important components of the primary health care system.

Similar to many other jurisdictions, South Africa has a large gap in pandemic response capacity, especially in the provision of personal protective equipment (PPE) due to a lack of financial, regulatory, or infrastructure resources (Blignaut, Nemutandani and Samaranayake, 2020). Many states delay the treatment of only emergency care performed. Patients who get dental care are only $10 \%$. Only (7\%) dentists argue that it should still do dental care regularly, but $59 \%$ of dentists think that they should do emergency dental care to prevent being infected with COVID-19.

It has been proven that some dentists are infected by COVID in Indonesia and Thailand. In Indonesia, 24 medical professionals, including six dentists, have died from COVID-19 (Suttagul and Sirichitra, 2020).

Oral health diseases have doubled in both developed and developing countries. It is of great concern that dental care services are inaccessible to most of the population due to the expensive nature of treatment modalities.

In most middle- and lower-income countries during the pandemic, the fact that dental care is poorly regulated and is not even considered an important component of the primary health care system. In addition, the question arises whether dental health care workers will comply with infection prevention and control during practice (Pethani, Allana and Hussain, 2020).

In middle- and lower-income countries with limited medical staff and a large range of dental health care, it is important to take appropriate measures in accordance with infection prevention and control protocols (Pethani, Allana and Hussain, 2020). When carrying out treatment measures should pay attention to the control of the spread of infection. Identify risk factors associated with infection and then take appropriate measures to reduce the risk of infection (El-Sokkary et al., 2021).

There are practical guidelines recommended for dentists and dental staff by the Centers for Disease Control and Prevention (CDC), the American Dental Association (ADA), and the World Health Organization to control the spread of COVID-19. Like other infectious infections, these recommendations include personal protective equipment, hand washing, detailed patient evaluation, rubber dam isolation, gargling before dental treatment procedures, and disinfection of the clinic. In addition, several guidelines and reports have provided useful information about the signs and symptoms of the disease, ways of 
transmission, and referral mechanisms to improve dentist knowledge and prevention practices, so as to contribute, at the population level, in disease control and prevention (Khader et al., 2020).

Due to the high risk of cross-infection in dental services, between dental and patient health workers, it is important to implement strict and effective infection control protocols in dental practice in middle and lower income countries. This literature review aims to provide knowledge about the strategies of middle and lower income countries in conducting infection prevention and control practices during the Covid-19 pandemic.

\section{Research Methods}

\subsection{Search Technique}

The literature review in this study was passed through systematic selection that was traced from an international database. The author searches data sources from various databases, namely, using PubMed (https://www.ncbi.nlm.nih.gov/pubmed/) and Mendeley (https://www.mendeley.com/). literature search technique using keywords that match the questions from the research.

Search for articles in English and published in English, the publication year is limited to the last 2 years (2019-2021), to be precise until May 9, 2021. The search strategy for Pubmed database uses AND and OR and signs ", while Mendeley uses AND and signs “.

\subsection{Article Inclusion and Exclusion Criteria}

This study aims to study related strategies for prevention and infection control of dental health service practices during the Covid-19 pandemic in developing countries. The inclusion criteria used to determine articles worthy of inclusion in the review are as follows:

1. The research article was conducted in middle- and lower-income countries. Criteria for income countries to top the list of worldbanks 2021 (The world bank, 2021)

2. Research article discussing infection prevention and control strategies in dentistry practice during the covid19 pandemic

3. Articles taken from 2019 to 2021

The article exclusion criteria were as follows:

1. Articles that are editorial

2. Full text is not in English

\subsection{Article Selection Method}

Articles are subjected to a relevant screening process, then analyzed according to the inclusion and exclusion criteria of research articles. Articles are analyzed according to the review topic, objectives and questions in the review. The selection process starts from the title, the abstract, is reviewed in full text, until later it is included in the article. Then, the article is seen whether it meets the inclusion criteria or not. 


\subsection{Method of Organizing Articles}

Based on the stages of the PRISMA diagram, the role of the first researcher is to identify articles through various databases of Data Collection Plans / Matrices. Organizing the findings articles in the study, researchers used Zotero (bibliographic software). All articles are analyzed and filtered for relevance and quality. Researchers created a special folder on zotero with the name according to the database name used in the search for articles. The researcher saves the articles obtained, then screens whether there are duplicated articles from each database. The duplicated article is then issued. Furthermore, the researcher screened the title and abstract whether it was relevant or not so that the article was relevant and worthy of being included in the next stage. Researchers read the entire text of the articles that have been deemed relevant and feasible and then analyzed whether the articles met the inclusion and exclusion criteria in the study.

\section{Research Results And Discussion}

\subsection{Research Results}

Figure 1 illustrates the article selection process using guidelines from Preferred Reporting Systematic Reviews and Meta-analysis (PRISMA). In the initial search, the number of articles from 20192021 was 2,483 articles. Then duplicates were excluded $(n=26)$. The article was screened $(n=2457)$. A total of 13 articles were submitted to the next stage.

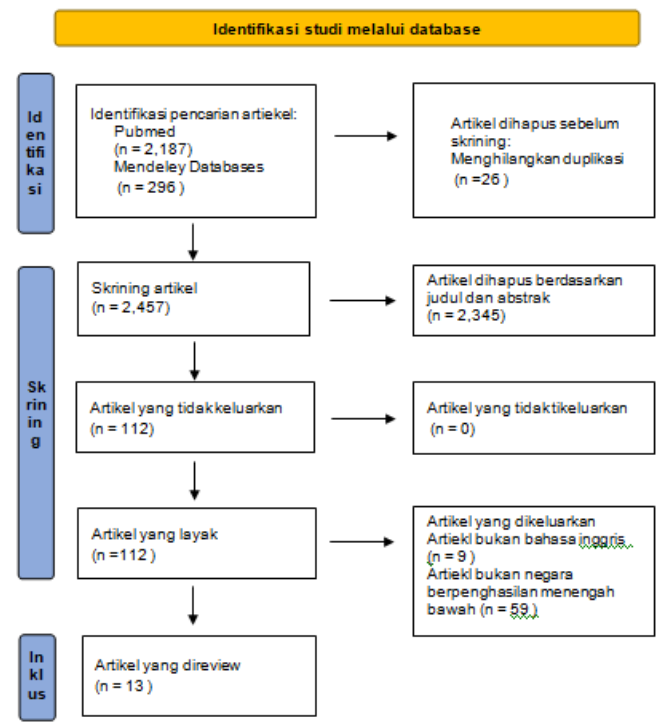

Figure 1. PRISMA Diagram

\subsection{Discussion}

The results of the review article show that the strategies for preventing and controlling infection in dental health care practices during the Covid-19 pandemic in middle- and lower-income countries in several aspects, namely patient screening, pre-treatment, treatment, post-treatment in dental patient care during the Covid-19 pandemic and setting up dental health service practice rooms. 
The impact of COVID-19 that has been reported in dentistry is a decrease in the number of patients. Only $38 \%$ of patients visit dental practices during the COVID-19 pandemic. Dental health cases dominated by dental trauma and oral infections.

COVID-19 has been considered a new pandemic in the world. This disease is highly contagious and can be transmitted easily through droplets and the air. It is considered a threat to all dentists around the world. Currently there are only a few specific guidelines with regard to dental practice during and after the pandemic, not many guidelines in countries or regions with limitations. Recommendations are needed based on the literature evidence on infection control because there is no single protocol that can fully guarantee patient and staff safety, especially for areas with various limitations.

During the adaptation period of new habits, dental and oral health services can be performed after careful consideration of the patient's condition and the risks faced by both patients and dental health care personnel, adjusting the availability of personal protective equipment (PPE) and other PPI support facilities as well as the rate of spread of COVID-19 infection in the local community. In countries with lower middle incomes found problems of PPE limitations and infrastructure facilities in dental and oral health services practices, dental and oral health services are prioritized only for emergency patients (Evans, 2020).

During the COVID-19 pandemic, dentists should limit treatment procedures during practice and reduce the number of patient visits. During the COVID-19 pandemic, some protocols require keeping a distance, limiting interpersonal contactl and reduce patient queues in waiting rooms. Dentists are recommended to use level 3 personal protective equipment when performing emergency treatment. It is also recommended to postpone all elective treatments for an indefinite period of time (Hudyono et al., 2020).

Patients receiving dental care are only $10 \%$ of dentists. It has been proven that some dentists are infected with the COVID-19 virus. Infection prevention and control during practice should be evaluated, given appropriate protective and control measures (Suttagul and Sirichitra, 2020). To address the new 'normal' stating that dentistry must 'adapt and adopt' to provide care to patients, especially in middle and lower income countries so dental healthcare workers should take appropriate precautions to avoid crossinfection. In dental health practice, the most common splashes occur in the nose and inner eye. The Indonesian Dental Association has raised specific concerns for PPE which include wearing and doffing PPE, as well as handwashing techniques, according to who (Hudyono et al., 2020).

Until now, dental healthcare practice guidelines have reopened with limited care. Indonesia has a tropical climate, the average daily temperature is hot with relatively high humidity. Infection prevention and protection guidelines in Indonesia are divided into Personal Protective Equipment (PPE) and hand hygiene protects dentists, dental teams, and also patients.

Infection prevention and control efforts in middle and lower income countries require a readaptation to infection control guidelines that can be easily applied in dental health care practices (Blignaut, Nemutandani and Samaranayake, 2020).

Dentists in lower-middle-income countries such as India experience high levels of anxiety and fear while practicing. For low-income countries, the most thorough temperature or triage measurements 
are performed to prevent possible SARS-CoV-2 virus infection, but cannot guarantee a patient is not infected (Blignaut, Nemutandani and Samaranayake, 2020). The best way to prevent COVID-19 is to get complete information about the COVID-19 virus and its transmission.

\section{Patient Screening}

For low-income countries, the most thorough temperature measurement or triage is carried out to prevent the possibility of infection with the SARS-CoV-2 virus, but it cannot guarantee a patient is not infected but screening and triage of patients is the best way to prevent COVID-19 with information complete information about the COVID-19 virus on the patient's condition(Blignaut, Nemutandani and Samaranayake, 2020).

The screening officer at the reception must be highly dedicated to screening and triage of patients. The things that are needed at the reception desk are thermometers and oximetry.A simple, inexpensive, reliable, non-contact electronic thermometer (at least two) should be available to check the temperature of all patients, the person delivering (if any), and all clinic staff and administrative staff at the entrance to the clinic (Blignaut, Nemutandani and Samaranayake, 2020). Temperature measurement cannot be used to indicate a person is not infected with COVID-19 due to asymptomatic symptoms. To prevent the spread of infection the patient should be instructed to wear a mask and cover the mouth and nose when coughing or sneezing. The patient's discrining assistant should be highly dedicated to screening and triage the patient(Suttagul and Sirichitra, 2020).

For visiting patients, it is mandatory to wear a mask. Scarcity of respiratory protective equipment, especially facial mask. This scarcity can be overcome by local production) cloth masks (maximum three layers) that can be washed, sterilized and reused, according to standard guidelines (Blignaut, Nemutandani and Samaranayake, 2020).

Reduce patient waiting times and ask patients to reduce the number of people accompanying them (Karnataka et al., 2020). Ask the patient to arrive on time as planned. Scheduling a patient visit plan can minimize the possibility of contact with other patients in waiting indoors (Wang et al., 2020).

Removing items from dental clinics (magazines, newspapers, and other objects) to reduce viral contamination (Rokaya, 2020). Applying teledentistry to triage patients by phone and knowing dental health problems for early screening of patient cases can also be done to reduce contact and reduce queues (Wang et al., 2020).

If a patient is diagnosed with Covid-19, the patient arrives in an empty waiting room, if possible they should be seated in a separate dental chair from other patients, in a well-ventilated room. Ideally, infected patients should not be treated in the clinical practice room, but only in AIIR (AirborneInfection Isolation Rooms) (Dacic, Miljkovic and Jovanovic, 2020).

The Indonesian Dental Association has adopted several guidelines for the treatment that should be taken during a pandemic. it is advisable to postpone elective treatment and undertake telemedicine. Telemedicine can be used to take anamnesis, visual examination, make a diagnosis and even prescribe medication.

Patient triage(Cheong, 2020): 
- Do you have the flu or flu-like symptoms such as fever with a temperature of more than $37.5^{\circ}$ $\mathrm{C}$, cough, sore throat, runny nose, shortness of breath, wheezing, headache, fatigue, muscle aches and chills sudden onset?

- Have you ever travelled abroad or to one of the red zone area places in the last fourteen days?

- Have you ever been in contact with someone confirmed with COVID-19/anyone under quarantine/person under investigation.

\section{Pre-treatment}

PDental health service personnel must follow guidelines from who or CDC, including personal protective equipment (PPE) and hand hygiene (Suttagul and Sirichitra, 2020). Doing hand washing before wearing the mask and donning procedure (Blignaut, Nemutandani and Samaranayake, 2020).

Hand hygiene is also important in dental health care practices. WHO always recommends washing your hands. WHO always recommends washing your hands in health facilities. Use clean and running water to wash your hands. Use soap to taste and apply covering all surfaces of the hands. Wash hands for 40-60 seconds enough to clean all surfaces. The use of disposable towels is recommended (Hudyono et al., 2020).

Use PPE based on the risks associated with the procedure. Risk assessment (Dacic, Miljkovic and Jovanovic, 2020):

- very high risk exposure perform procedures that produce aerosols for a long / short time, without the use of PPE

- $\quad$ high risk exposure perform procedures that produce aerosols for a long / short time, with the use of PPE.

- medium risk exposure - perform procedures that produce non-aerosols for a long time, with the use of PPE.

- low risk exposure 
Perform urgent procedures and do not cause aerosols for a short time, with the use of PPE.

Personal protection equipment according to the risk (Wang et al., 2020)

Table 1. Personal protection equipment according to the risk

\begin{tabular}{lll}
\hline Officer & Personal Protection Equipment (PPE) \\
\hline Screening/triage officer & $\begin{array}{l}\text { surgical caps, eye protection, face protectors, } \\
\text { surgical masks. }\end{array}$ \\
\hline Disinfection officer & gloves, insulating gown, plastic shoe cover \\
\hline $\begin{array}{l}\text { Dentists and nurses in the } \\
\text { treatment room }\end{array}$ & $\begin{array}{l}\text { Surgical cap, eye protection, face protection, } \\
\text { surgical mask, gloves, isolation gown, plastic } \\
\text { shoe cover }\end{array}$ \\
\hline $\begin{array}{l}\text { Dentists and nurses in } \\
\text { aerosol action }\end{array}$ & $\begin{array}{l}\text { Surgical cap, eye protection, face protection, } \\
\text { surgical mask (+ N95 respiratory shield), } \\
\text { gloves, isolation gown, plastic shoe cover }\end{array}$ \\
\hline $\begin{array}{l}\text { Dentists and nurses in } \\
\text { aerosol action }\end{array}$ & $\begin{array}{l}\text { Surgical cap, eye protection, face protection, } \\
\text { surgical mask (+ N95 respiratory shield), }\end{array}$ \\
\hline
\end{tabular}

Use of masks N95 (United States NIOSH-42CFR84), FFP2 or FFP3 (European EN 149-2001),

KN95 (China GB2626-2006 recommended because of their proper size and superior filtration ( $>95 \%)$ of small droplets in the air (particle 1) - $\mu \mathrm{m}$ ) (Otieno, Kihara and Mua, 2020). This device is disposable and is used on the face, covering the nose and mouth. A number of studies have been conducted to test the effectiveness of surgical masks and N95 respirators in protecting the wearer, especially from virus infections. Although N95 respirators offer a high degree of protection, they can inhibit air exchange and thereby increase the metabolic workload. Dentists should be warned about the negative effects of prolonged N95 use. It is possible to cause discomfort, tightness due to increased respiratory air resistance and CO2 levels. Some wearers also complain of headaches, dizziness, and difficulty communicating (Hudyono et al., 2020).

In some cases in countries with a scarcity of PPE, this can be circumvented by reusing N95 masks. If there is a shortage of PPE, especially masks, the CDC recommends hanging and drying masks or storing masks in closed containers with ventilation holes, at each end of use provided that the masks are in good condition and the outer surface of the mask does not come into contact / minimal contact with contaminants / pathogenic microorganisms. The use of this mask must be worn by the same person, not used interchangeably. It should be noted, users must be careful when removing (doffing) the mask so that the inside is not touched/contaminated, as well as when donning again. Reuse masks are only recommended for a maximum of 3-5 times of use.

If disposable gowns are not available, they can be made using a large, white, thin plastic bag by cutting holes at the top, for the head, and on the sides. Plastic gowns can protect clothes from splashes and splashes. Such materials can be disposed of aseptically after each procedure (Blignaut, Nemutandani and Samaranayake, 2020).

face shields should be used to reduce aerosol exposure. Splashes from treatment procedures may occur so eye protection is mandatory

The following is the procedure for donning / wearing PPE according to Cheong (2020) 


\begin{tabular}{|c|c|c|}
\hline Level 2 (Droplet) & Level 2 (Airborne) & Level 3 \\
\hline Hand wash \& hand rub & Hand wash \& hand rub & Hand wash \& hand rub \\
\hline$\downarrow$ & $\downarrow$ & $\downarrow$ \\
\hline Surgical mask & N95 mask & N95 mask \\
\hline$\downarrow$ & $\downarrow$ & $\downarrow$ \\
\hline Head cover & Head cover & Jumpsuit \\
\hline$\downarrow$ & $\downarrow$ & $\downarrow$ \\
\hline Shoes cover & Shoes cover & Shoes cover \\
\hline$\downarrow$ & $\downarrow$ & $\downarrow$ \\
\hline Hand rub & Hand rub & Hand rub \\
\hline$\downarrow$ & $\downarrow$ & $\downarrow$ \\
\hline $1^{\text {st }}$ layer of glove & $1^{\text {st }}$ layer of glove & Plastic apron / Gown \\
\hline$\downarrow$ & $\downarrow$ & $\downarrow$ \\
\hline Full body gown & Full body gown & $1^{\text {st }}$ layer of glove \\
\hline$\downarrow$ & $\downarrow$ & $\downarrow$ \\
\hline Plastic apron & Plastic apron & $2^{\text {nd }}$ layer of glove \\
\hline$\downarrow$ & $\downarrow$ & $\downarrow$ \\
\hline $2^{\text {nd }}$ layer of glove & $2^{\text {nd }}$ layer of glove & Face shield \\
\hline$\downarrow$ & $\downarrow$ & \\
\hline Face shield & Face shield & \\
\hline
\end{tabular}

Figure 2. The procedure for donning / wearing PPE (Cheong, 2020)

Second screening before treatment (Rokaya, 2020):

- Emergency care.

- Urgent conditions: treated with minimally invasive procedures without producing aerosols or being treated with invasive and / or aerosol generation

- Nonroutine care.

- Routine care

Then the patient was instructed to use mouthwash containing povidone-iodine $0.2 \%$ or $0.5-1 \%$ hydrogen peroxide because this antiseptic has been shown to have virucidal activity and reduce the percentage of Coronavirus in his saliva before performing dental treatment procedures (Dacic, Miljkovic and Jovanovic, 2020).

Selection of patients to reduce the possibility of transmission of COVID-19 during dental treatment is recommended to reduce the action that produces aerosols, when the use is unavoidable it should reduce aerosols that spread throughout the room and disinfect the room, after treatment. Thus cross infection or spread of infection will not occur (Hudyono et al., 2020).

\section{Treatment}

Dentists need to deal with various dental emergencies during the COVID-19 pandemic. Emergency dental care is needed for dental trauma and oral infections. Irreversible symptomatic pulpitis is the most common during the COVID-19 pandemic. Emergency case, vital pulp therapy can be performed, reduce the treatment time and reduce the risk of further infection. Pharmacological management (antibiotic and/or analgesics) can be an alternative as long as COVID-19 Patients can be referred to a more likely place for further treatment (Ghani, 2020).

There are areas with the availability of infrastructure facilities less supportive of services, delay emergency and elective case care for a period of 2-3 weeks, especially for patients with probabel status and confirmed positive COVID-19 (Lee et al., 2020). 
The duration of patient visits should be as short as possible. Treatment procedures performed should be as minimally invasive as possible and avoid or reduce aerosol-generating procedures with handpieces and uss scalers. Use single-use equipment whenever possible to limit cross-infection or crosscontamination.

Category Risk Level of Work and Treatment in Dental and Oral Health Services: Low :

- Not in direct contact with patients

- Not exposed to droplets and aerosols

- Keep social distance of officers and patients

Medium :

- Close contact with healthy/non-COVID-19 patients when performing emergency and urgent case services,

- Not exposed to aerosols

- Public dental and oral health services with the application of health protocols.

High :

- Close contact and non-aerosol actions, in patients with COVID-19 confirmed

- Close contact and aerosol action in healthy/ non-COVID-19 patients

When practicing dental health care workers should be aware of the following (Ghani, 2020).:

- Dental practitioners must follow guidelines from the WHO or CDC, including personal protective equipment (PPE) and hand hygiene. It is recommended to use the N95 mark and reusable bias occurs scarcity of PPE.

- Patients gargle with mouthwash containing povidone iodine $0.2 \%$ or $0.5-1 \%$ hydrogen peroxide to help reduce the spread of the covid-19 virus.

- The use of disposable instruments such as mouth glass, diagnostic probes, and syringes can be used to prevent

- Can be used rubber dam (covering nose) to minimize the formation of aerosol splashes.

- Reduces the use of ultrasonic instruments, high-speed handpieces, and try way syrings to prevent contamination from aerosols

- Suspected dental care or confirmed COVID-19 patients should be performed in the room (AIIR) or negative pressure treatment

- Good knowledge and positive attitude of health care staff towards COVID-19 and AIIR room can assist dentists in providing dental care and treatment. If there is no AIIR space, in certain regions it can be referred to other health facilities.

- SARS CoV-2 can survive in the air for about 3 days so the surface must be disinfected using chemicals to prevent the spread of the virus. 
All necessary materials and instruments must be prepared in advance to make the procedure faster. Disposable protection should be placed on the surface of the unit and disposable instruments should be used at all times, if possible (Negucioiu et al., 2020). Few dentists use disposable shields because of the added cost. Currently, instrument and personal protective materials are recommended as protection for barrier surfaces and equipment that are difficult to decontaminate(Otieno, Kihara and Mua, 2020).

The duration of the patient's visit should be as short as possible. Treatment procedures performed should be minimally invasive and avoid or reduce aerosol-generating measures with the handpiece and USS scaller. Use disposable tools whenever possible to limit cross infection or cross contamination. performing procedures with aerosols, the use of surgical masks alone is not sufficient, a particulate respirator with a high-efficiency particulate air (HEPA) filter is required (Dacic, Miljkovic and Jovanovic, 2020). Rubber dam isolation combined with high volume vacuum dental aerosol is required to control the spread of microorganisms via sprinkling and bioaerosol. Intraoral radiographs should be limited to reduce saliva and vomiting reflex instead of panoramic radiogafi (Varma et al., 2020).

\section{Post-Treatment}

The patient is asked to put the mask back on and take off his protection. Staff cleaning and disinfecting the work environment. Pause the patient at least 5 minutes to change the air circulation. Using disposable tools protection.

Only a few dentists use disposable protectors because they are economical and feel that they provide protection from dental surfaces, equipment, and materials. Currently, instrument protection materials and personal protection are recommended as protection for surface barriers and equipment that is difficult to decontaminate. Cleaning can be done at the end of the practice. A barrier is placed between the patient. If a dirty surface is found it requires immediate decontamination (Otieno, Kihara and Mua, 2020).

If there is a shortage of PPE, especially masks, the CDC recommends hanging and drying masks or storing masks in a closed container with ventilation holes, after each use provided that the mask is in good condition and the outer surface of the mask has no minimal contact with pathogenic contaminants / microorganisms. The use of this mask must be worn by the same person, not used interchangeably. It should be noted, users must be careful when removing (doffing) the mask so that the inside is not touched / contaminated, as well as when putting (donning) back. Reuse masks are only recommended for a maximum of 3-5 times of use.

Instruments classified as critical require sterilization or single use due to contact with sterile tissue, while semicritical instruments can be sterilized or disinfected with high-level disinfectants. The dental hygienist disinfects the dental chair after each patient. A review of the literature shows that previously known coronavirus persists on surfaces for up to 9 days, but disinfection of surfaces with $0.1 \%$ sodium hypochlorite or $62-71 \%$ ethanol can inactivate the virus (Otieno, Kihara and Mua, 2020).

Decontamination classification based on the type of goods contaminated according to the CDC: 
Critical: objects that present a high risk of infection if contaminated with microorganisms. surgical instruments, implants, and ultrasonic probes (scalers, handpieces, bone burs, probes etc.). Ways of decontamination with sterilization.

Semi-critical: objects that have come into contact with the mucosa or skin that are not intact (there is a need or contact in the mucosa). Printed spoon instruments, handpiece bur, dental diagnostic tools, the edge of bur. Decontamination by disinfection using high-level disinfectants.

Non-critical: objects that have come into contact with intact skin but not mucosa. Care items for noncritical patients; items found on non-critical environmental surfaces such as blood pressure gauge cuffs, stethoscopes and computers). Disinfection using disinfectants containing detergents or alcohol.Wipe with disinfectant wipes containing $75 \%$ alcohol or $1,000 \mathrm{mg} / \mathrm{L}$ chlorine disinfectant solution after each procedure.

Decontamination of the room can use air aerosol spray with $10 \%$ sodium hypochlorite solution, ultraviolet disinfection lamp for 30 minutes. Mopping the floor with a chlorine disinfectant solution of $1,000 \mathrm{mg} / \mathrm{L}$ Two 2 times a day.

UV rays are currently used to disinfect water, air and surfaces. Disinfection of surfaces is limited due to shading or protective coating. Several studies have stated that UV is effective in deactivating coronavirus in dental practice (Hudyono et al., 2020). This can be applied to reduce contamination in the air and reduce costs because it is relatively cheap.

Proper handling and disposal of clinical waste plays an important role in controlling the spread of disease and loss to the community. Prevention and control guidelines for health care services in Kenya recommend burning infectious waste. Further studies should be conducted to assess the level of knowledge and compliance with local policies on waste management. The separation of sharp objects in specially labeled areas should be carried out by all dental practices. Therefore, further training and development of special protocols for the safety of sharp objects is required (Otieno, Kihara and Mua, 2020).

\section{Dental Health Practice Room}

Middle and lower income countries or developing countries are encouraged to open all windows as much as possible in practice rooms and entrance areas for waiting rooms (Blignaut, Nemutandani and Samaranayake, 2020). If possible, provide an outpatient care room once every 30 minutes, to provide room for adequate surface disinfection and ventilation, although this may not allow busy clinics. Posters on hand washing and wearing of masks and doffing procedures, and those related to the spread of COVID-19, should be prominently displayed in the local language in the reception area.

In areas with hot weather, room conditioner is needed in every dental practice. However, these devices make it difficult to disinfect the room and can cause the virus to spread throughout the room. For room disinfection, prepare the conditions for ventilation of air, ozone and hydroxyl agents and cleaning the floors and surfaces of the dental unit. The importance of air-ventilated spaces in dental practice is well explained in several studies. One study found that high levels of $\mathrm{CO} 2$, total VOC and particulate matter were found during operating hours, and significantly reduced during closing hours. Ventilation levels are measured as hourly air changes ( $\mathrm{ACH}$, sometimes abbreviated as $\mathrm{ACPH})$. It is a measure of how much 
volume of air is changed, either added or eliminated, from a room in an hour. Measuring the number of times air in a room is changed to achieve equilibrium pressure, the amount of air that comes out and enters the room must be the same. ACH is measured by dividing the change in airflow in a room for an hour by the volume of the room itself (Hudyono et al., 2020).

The level of air ventilation, aerosol control of dispersal, and disinfection of floor surfaces and dental units are beneficial in reducing indoor contaminants. Until now there is no protocol that can be used to sterilize air conditioning in dental practice facilities. Ozone may be promising, but it needs further research. We recommend limiting the use of air conditioning units as little as possible. air conditioning unit in the waiting room, and let the cool air enter the negative pressure room of the exhaust fan. Barriers to space made with plastic can help maintain distance and ease decontamination, this becomes an alternative in countries with lower middle incomes (Hudyono et al., 2020).

Aerosol Suction and High Vacuum Evacuator are central vacuum systems with 28.5 liters per minute effective in reducing aerosols by $36 \%$. The vacuum system is reported to be more effective in reducing aerosol exposure in dentists who use only filtering respirators. Another study also found that high-vacuum evacuators could reduce the exposure of patients and dentists to very fine particles by more than $80 \%$ compared to conventional suction teeth. Some dentists also modify the vacuum cleaner to become an aerosol suction device(Dee et al., 2006).

Air ventilation rates, control of aerosol spread and disinfection of floor surfaces and dental units are beneficial in reducing indoor contaminants. Until now, there is no protocol that can be used to sterilize air conditioners in dental practice facilities. Ozone is thought to be able to sterilize air conditioners, but needs further investigation. The protocol recommends limiting the use of AC units to as little as possible. In the waiting room let cool air enter the room and negative pressure from the exhaust fan. Space barriers can be made with plastic, which can help to keep the distance and easy to decontaminate, this is an alternative in lower-middle income countries to prevent contact and spread of infection(Hudyono et al., 2020)

During the COVID-19 pandemic, this requirement was incorporated into dental clinic management. The semi-polluted area is a buffer zone from the clean area to the dental treatment area, where dentists and nurses carry out hand washing, disinfection, and change their shoes and clothes before moving from one area to another. Due to limited equipment and practice layouts it is possible not to achieve positive air pressure in the clean zone, or negative air pressure in the semi-polluted and patient zones, the air pressure of the three zones is the same. Central air conditioning and ventilation systems are turned off to avoid cross-infection between zones or even throughout the building. The aerosol-generating action room is isolated and located away from other rooms isolated treatment rooms for patients in high risk of COVID-19. Isolated treatment rooms are prepared for patients who have been evaluated as at high risk of COVID-19, and simultaneously require emergency oral care. Disinfection of the environment will be stricter and no further treatment will be carried out for at least 4 hours (Wang et al., 2020).

The waiting room is plastered with posters about hand hygiene, cough etiquette, and face mask coverage during the visit period should be displayed in the waiting room. The number of patients should be appropriate in the waiting room and a remote seat should also be considered. Disinfection of the 
waiting room is also carried out thoroughly on potentially contaminated surfaces as doorknobs, chairs and tables are also highly recommended.

Hand sanitizer/hand soap in front of the entrance. The furniture and interior of the dental clinic should be assessed, and if necessary, rearranged to allow for a safe social distance. The route inside the dental clinic should be arranged in such a way that dental health service personnel and patients can keep their distance from each other. DHCW does not use PPE. The social distance between dental health services should also be maintained when not treating patients (Blignaut, Nemutandani and Samaranayake, 2020).

\section{Conclusion}

The findings article obtained 20 articles discussing infection prevention and control in dental health service practices during the Covid-19 pandemic in middle and lower income countries. Dentists need to deal with various dental health diseases during the COVID-19 pandemic.

COVID-19 has an impact on dental health service practices. The dental health service practitioner must excavate and assess the condition of patients who come for dental treatment. Prevention and control measures for infection are required in dental service practices in accordance with existing regulations. Cross infection can be minimized by getting complete information about the COVID-19 virus, its transmission and prevention methods. So that it can reduce cross infection in dental service practices. Dental procedures should be carried out with infection prevention and control following appropriate recommendations.

In middle and lower income countries, patient screening procedures are very important to determine the patient's health condition because the Covid test procedure and equipment are not optimal. Screening the patient's medical history and family members such as contact with a person infected with COVID-19, history of fever, and traveling in the last 14 days. The patient's temperature should be measured using a non-contact thermometer. The patient should be instructed to wear a mask and cover the mouth and nose when coughing or sneezing. Another alternative is to reduce patient queues by scheduling visits and asking patients to reduce the number of people accompanying them so as to create distance in the waiting room. Use of personal protective equipment (PPE), examination of patients, practice hand hygiene, use of single-use instruments, use rubber dams, reduce the use of aerosol-generating tools, select cases and treat patients with COVID-19 confirmation in a separate room. It is advisable to delay or reduce non-emergency measures (dental trauma and oral infections, symptomatic irreversible pulpitis, vital pulp therapy can be done to reduce treatment time and reduce the risk of further infection) if the practice room does not have a HEPA filter or lack of ventilation.

Surface disinfection and tool decontamination are required after dental hygiene measures. PPE change every change of patient and pause each procedure for 30 minutes for surface disinfection. In some areas there is a scarcity of PPE, especially N95 masks, which can be reused with proper decontamination. There is no need for a single-use instrument to reduce costs, but a decontamination method in accordance with the protocol. 
When dental health service workers do not wear PPE, dental health service workers must keep their distance, have good ventilation and keep a distance in the waiting room. The practical layout and waiting room are provided with hand sanitizers / hand washing soap in front of the entrance. Dental clinic furniture and interiors should be remanufactured to maintain a safe distance. All unnecessary furniture such as bookshelves, magazines and koans must be removed from the clinic to prevent viruses and bacteria from sticking. The grooves in the dental clinic must be arranged in such a way that dental health care workers and patients can maintain a distance from each other. A glass or plastic barrier is installed to reduce contact.

\section{Suggestion}

Dental health care workers must study and gather information related to infection prevention and control protocols. Staff who understand infection prevention and control are needed to prevent crossinfection or spread of infection in dental practice. The role of dental service officers in middle and lower income countries must be smart in adopting and modifying infection prevention and control protocols from all aspects that can be applied in practice so as to reduce the spread of Covid-19 infections.

\section{References}

Ahmed, S. et al. (2021) 'Awareness and Practice of Infection Control Protocol Amid Covid-19 Pandemi in Private Dental Clinics in Saudi Arabia', Journal of Pharmaceutical Research International, pp. 1-7. doi: 10.9734/jpri/2020/v32i4231048.

Blignaut, E., Nemutandani, S. and Samaranayake, L. (2020) 'Strategic implementation of dental infection control in resource-poor jurisdictions during COVID-19', ACTA ODONTOLOGICA SCANDINAVICA, p. 5.

Blignaut, E., Nemutandani, S. and Samaranayake, L. (2020) 'Strategic implementation of dental infection control in resource-poor jurisdictions during COVID-19 pandemi: a perspective from South Africa', Acta Odontologica Scandinavica, 78(8), pp. 614617. doi: $10.1080 / 00016357.2020 .1804073$.

Cheong, J. M. (2020) 'Safe dental practice during the COVID-19 pandemi', IIUM Journal of Orofacial and Health Sciences, 1(2), pp. 106-120. doi: $10.31436 /$ ijohs.v1i2.56.

Dacic, S. D., Miljkovic, M. N. and Jovanovic, M. C. (2020) 'Dental care during the Covid-19 pandemi - To treat or not to treat?', The Journal of Infection in Developing Countries, 14(10), pp. 1111-1116. doi: 10.3855/jidc.13147.

Dee, S. A. et al. (2006) 'Further evaluation of alternative air-filtration systems for reducing the transmission of Porcine reproductive and respiratory syndrome virus by aerosol', Canadian Journal of Veterinary Research, 70(3), pp. 168-175.

Diksha Agrawal, Priyanka Jaiswal, and Pavan Bajaj (2020) 'Role of Dentist to Stop COVID-19: A Journey map', International Journal of Research in Pharmaceutical Sciences, 11(SPL1), pp. 880-884. doi: 10.26452/ijrps.v11iSPL1.3109.

El-Sokkary, R. H. et al. (2021) 'Characteristics and predicting factors of Corona Virus Disease-2019 (COVID-19) among healthcare providers in a developing country’, PLOS ONE, 16(1). doi: $10.1371 /$ journal.pone. 0245672.

Epidemiological and clinical characteristics of 99 cases of 2019 novel coronavirus pneumonia in Wuhan, China: a descriptive study (no date). Available at: https://www.ncbi.nlm.nih.gov/pmc/articles/PMC7135076/ (Accessed: 20 April 2021).

Evans, G. (2020) 'CDC Updates Guidance on COVID-19 Transmission in Dental Settings: Concerns about aerosols, splashing.', Hospital Infection Control \& Prevention, 47(7), pp. 1-3.

Falahchai, M., Babaee Hemmati, Y. and Hasanzade, M. (2020) 'Dental care management during the COVID-19 outbreak', Special Care in Dentistry, 40(6), pp. 539-548. doi: $10.1111 /$ scd.12523.

Ghani, F. (2020) 'COVID-19 Outbreak and Dentistry: Guidelines and Recommendations for the Provision of Dental Healthcare Services', Journal of the College of Physicians and Surgeons Pakistan, 30(2), pp. 101-105. doi: $10.29271 /$ jepsp.2020.Supp2.101.

Hudyono, R. et al. (2020) 'During and post COVID-19 pandemi: prevention of cross infection at dental practices in country with tropical climate', Dental Journal (Majalah Kedokteran Gigi), 53(2), p. 81. doi: 10.20473/j.djmkg.v53.i2.p81-87.

Iurcov, R. et al. (2021) 'Evaluating the Practice of Preventive Behaviors and the Fear of COVID-19 among Dentists in Oradea Metropolitan Area after the First Wave of Pandemi; a Cross-Sectional Study', Healthcare (Basel, Switzerland), 9(4). doi: $10.3390 /$ healthcare9040443.

Khader, Y. et al. (2020) 'Dentists' Awareness, Perception, and Attitude Regarding COVID-19 and Infection Control: CrossSectional Study Among Jordanian Dentists', JMIR public health and surveillance, 6(2), p. e18798. doi: 10.2196/18798.

Lee, Y.-L. et al. (2020) 'Dental care and infection-control procedures during the COVID-19 pandemi: The experience in Taipei City Hospital, Taiwan', Journal of Dental Sciences, 15(3), pp. 369-372. doi: 10.1016/j.jds.2020.05.011.

Long, R. H. et al. (2020) 'Modifications of emergency dental clinic protocols to combat COVID-19 transmission', Special Care in Dentistry, 40(3), pp. 219-226. doi: $10.1111 /$ scd.12472.

Luzzi, V. et al. (2020) 'Paediatric Oral Health during and after the COVID-19 Pandemi', p. 7.

Mahase, E. (2020) 'China coronavirus: WHO declares international emergency as death toll exceeds 200', BMJ (Clinical research ed.), 368, p. m408. doi: 10.1136/bmj.m408.

Manjeet, R. et al. (2020) 'INFECTION CONTROL IN DENTAL CLINICS DURING COVID 19 -Original Review', Journal of Indian Dental Association, p. 25811. doi: 10.33882/jida.14.25811. 
McMahon, D. E. et al. (2020) 'Global resource shortages during COVID-19: Bad news for low-income countries', PLoS neglected tropical diseases, 14(7), p. e0008412. doi: 10.1371/journal.pntd.0008412.

Negucioiu, M. et al. (2020) 'Management of SARS-CoV-2 Transmission in Emergency Dental Settings: Current Knowledge and Personal Experience', Disaster Medicine and Public Health Preparedness, pp. 1-8. doi: 10.1017/dmp.2020.483.

Otieno, B. O., Kihara, E. N. and Mua, B. N. (2020) 'Infection Control Practices Among Private Practicing Dentists in Nairobi During the Pre-coronavirus Disease 2019 Period', Frontiers in Oral Health, 1, p. 587603. doi: 10.3389/froh.2020.587603.

Peng, X. et al. (2020) 'Transmission routes of 2019-nCoV and controls in dental practice', International Journal of Oral Science, 12(1), p. 9. doi: $10.1038 / \mathrm{s} 41368-020-0075-9$.

Pethani, A. S., Allana, R. R. and Hussain, M. (2020) 'Emerging Challenges and Threats for Dental Health Care Sector Attributable to COVID-19: Tale of a Developing Country', Asia-Pacific Journal of Public Health, 32(5), pp. 278-280. doi: $10.1177 / 1010539520932708$.

PG Student, Department of Paediatric and Preventive Dentistry, Bangalore Institute of Dental Sciences and Research Centre, Bangalore, Karnataka, India. et al. (2020) 'AWARENESS AMONGST DENTAL PRACTITIONERS REGARDING THE CHANGE IN INFECTION CONTROL PROTOCOLS DURING COVID-19 PANDEMI', International Journal of Advanced Research, 8(9), pp. 1284-1288. doi: 10.21474/IJAR01/11786.

Pious, N., Mhatre, A. and Ingole, S. (2020) 'Management of Coronavirus disease 2019 in dentistry', Journal of Oral Research and Review, 12(2), p. 110. doi: 10.4103 /jorr.jorr 1320.

Prospero, E., Savini, S. and Annino, I. (2003) 'Microbial Aerosol Contamination of Dental Healthcare Workers' Faces and Other Surfaces in Dental Practice', Infection Control \&Hospital Epidemiology, 24(2), pp. 139-141. doi: 10.1086/502172.

Rokaya, D. (2020) 'COVID-19: Prosthodontic Challenges and Opportunities in Dental Practice', Journal of Advanced Oral Research, 11(2), pp. 113-116. doi: 10.1177/2320206820953966.

Roshan A, Jeevitha M, and Sridevi G (2020) 'Analysis of awareness of infection control in dental practice during the COVID-19 outbreak among dental practitioners in Tamil Nadu', International Journal of Research in Pharmaceutical Sciences, 11(SPL1), pp. 653-658. doi: 10.26452 ijirps.v11iSPL1.3062.

Suttagul, K. and Sirichitra, V. (2020) 'COVID-19 Pandemi in ASEAN Region and Implication in Dentistry', Open Access Macedonian Journal of Medical Sciences, 8(T1), pp. 465-470. doi: 10.3889/oamjms.2020.5453.

Varma, B. et al. (2020) 'Dental Care Implications in Coronavirus Disease-19 Scenario: Perspectives', The Journal of Contemporary Dental Practice, 21(8), pp. 935-941. doi: 10.5005/jp-journals-10024-2896.

Wang, C. et al. (2020) 'Emergency Management in a Dental Clinic During the Coronavirus Disease 2019 (COVID-19) Epidemic in Beijing', p. 9. 